\title{
PREFERENCE FACTORING FOR STOCHASTIC TREES
}

\section{GORDON HAZEN}

\author{
Northwestern University
}

August 1999

Stochastic trees are extensions of decision trees which facilitate the modeling of temporal uncertainties. Their primary application has been to medical treatment decisions. It is often convenient to present stochastic trees in factored form, allowing loosely coupled pieces of the model to be formulated and presented separately. In this paper, we show how the notion of factoring can be extended as well to preference components of the stochastic model. We examine updateable-state utility, a flexible class of expected utility models which permit stochastic trees to be rolled back much in the manner of decision trees. We show that preference summaries for updateable-state utility can be factored out of the stochastic tree. In addition, we examine utility decompositions which can arise when factors in a stochastic tree are treated as attributes in a multiattribute utility function.

$\mathrm{S}$ tochastic trees are graphical modeling tools which extend decision trees by allowing the explicit depiction of temporal uncertainty. They are especially well suited to modeling temporal issues in medical treatment decision analyses (Hazen 1992). My colleagues and I have constructed stochastic tree models for total hip replacement and knee replacement decision analyses (Chang, Pellissier and Hazen 1996;
Pellissier, Hazen and Chang 1996; Gottlob et al. 1996), and for breast cancer decision analysis (Hazen, Morrow and Venta 1998). We have investigated risk-sensitive utility rollback for stochastic trees (Hazen and Pellissier 1996), and have explored preference assessment techniques which are tailored to address risky temporal tradeoffs (Hazen, Hopp and Pellissier 1991; Pellissier and Hazen 1994). We have found the notion of factoring a stochastic tree (Hazen 1993) 
to be very useful for model formulation and presentation, and have developed software (available at the author's web site) with a graphical interface for formulating and rolling back factored stochastic trees. All stochastic tree illustrations in this article are screen captures from that software.

Formulating a stochastic tree in a factored fashion can greatly simplify the model construction process. For example, it is usually beneficial to factor out background mortality and consider the rest of the model separately (Hazen 1993, Hazen, Pellissier and Sounderpandian 1998). Further factoring is usually helpful as well, for example, to separate essentially independent processes such as drug side effects and disease progression. We give examples of stochastic tree factoring below.

In this paper, we show how the notion of factoring can be extended as well to preference components of the stochastic model. When features involving patient preference are factored out, the remaining stochastic model is often significantly simpler. We take up preference factoring in Section 2, where we examine updateable-state utility models. These arise from the state-trajectory preference summaries recently introduced by Hazen and Sounderpandian (1999), and extend the Markovian utility models introduced by Hazen and Pellissier (1996). The major result presented in Section 2 is that every updateable-state utility model is equivalent to a
Markovian utility model in a stochastic tree augmented by factors which record preference summary states. The latter are in this sense factored from the model.

Of course, the factoring process establishes a natural multiattribute structure to any stochastic tree model, and this suggests that preference decomposition issues be investigated. In Section 3 we examine utility independence and related conditions, and show how such assumptions can be used to decompose updateable-state utility models. We begin in the next section with a review of factoring stochastic trees.

\section{FACTORING STOCHASTIC TREES}

\section{Background}

In its simplest form, a stochastic tree is merely a continuous-time Markov chain unfolded into a tree structure, with possible chance or decision nodes added. Straight-line arcs emanate from chance or decision nodes, and arcs from chance nodes have attached probabilities, just as in a decision tree. Wavy arcs emanate from stochastic nodes, and denote transitions which take time to occur. Wavy arcs have transition rates attached. Figure 1 displays a simplified stochastic tree model of transient ischemic attack adapted from Matchar and Pauker (1986). 


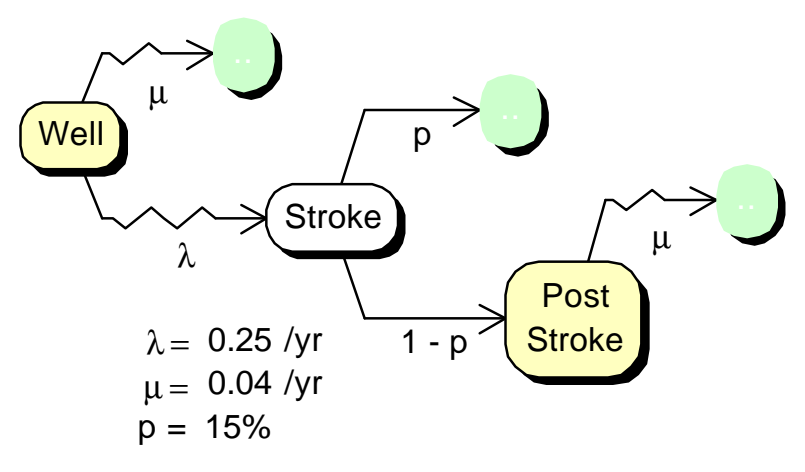

Figure 1: A stochastic tree model. Unlabeled shaded nodes represent death. A patient begins in the Well state with mortality risk due to unrelated causes occurring at average rate $\mu$, and competing risk of stroke occuring at average rate $\lambda$. Should death occur first, the process terminates. Should stroke occur first, the patient moves to the state Stroke, at which there is a chance $p$ of immediate mortality. Should the patient survive, he moves to the state Post Stroke, from which he is subject only to mortality risk at rate $\mu$.

Stochastic trees can be rolled back much like decision trees. Figure 2 illustrates how rollback can be used to calculate mean survival times. One may also calculate mean quality adjusted survival times, in which time spent in a state is weighted by its relative worth compared to an equal time in the well state. This is illustrated in Figure 3.

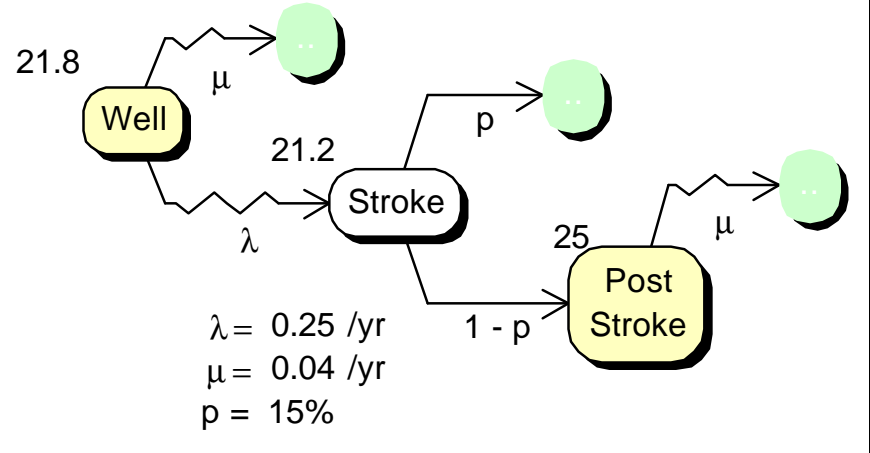

Figure 2: Using rollback to calculate mean survival duration in a stochastic tree. Numerical values at nodes are mean survival durations in years. For example, mean survival time at Post Stroke is $1 / \mu=25$ years, mean survival time at Stroke is $25 \times(1-p)=$ 21.2 years. Overall mean survival time is 21.8 years using the rollback formula (2) below.

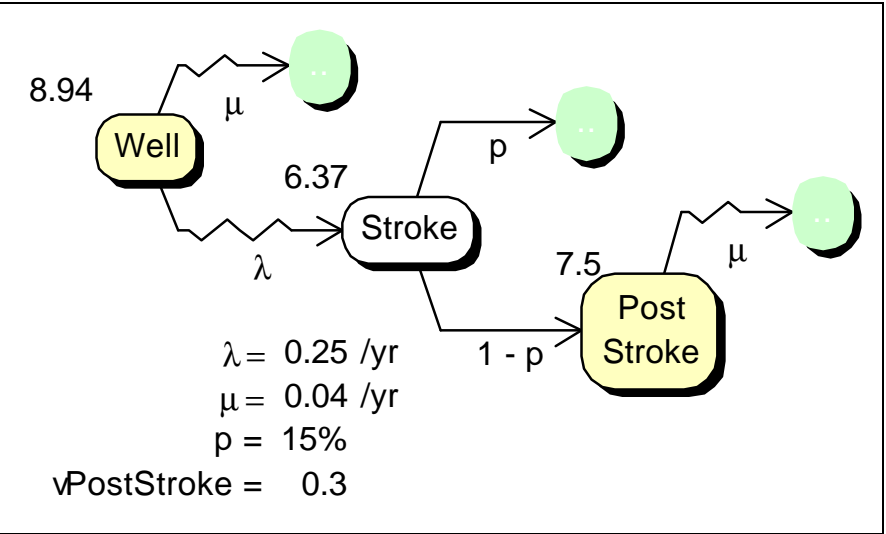

Figure 3: Using rollback to calculate mean quality adjusted lifetime. For example, mean quality-adjusted lifetime at Post Stroke is $(1 / \mu) \times v$ PostStroke $=7.5$ years, and mean survival time at Stroke is $7.5 \times(1-p)$ $=6.37$ years. Overall mean quality-adjusted lifetime is 8.94 years using the rollback formula (2) below

The generic rollback formula is given by Hazen (1992) or Hazen and Pellissier (1996). At an arbitrary stochastic fork 


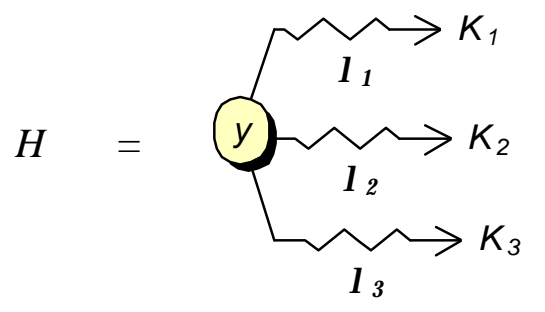

in which subtrees $K_{i}$ are reached from state $y$ at competing rates $\lambda_{i}$, the rollback equation for mean discounted quality adjusted lifetime $L(\cdot)$ takes the simple form

$$
L(H)=\frac{v(y)+\sum_{i} \lambda_{i} L\left(K_{i}\right)}{a(y)+\sum_{i} \lambda_{i}} .
$$

Here $v(y)$ is the quality weight for state $y$, and $a(y)$ is the state-specific discount rate at state $y$. When $a(y)=0$ for all $y$, expected utility is mean qualityadjusted duration, and when $a(y)=\alpha$ for all $y$, expected utility is quality-adjusted duration discounted at rate $\alpha$. At chance forks, expected utility computation is identical to the usual probability-weighted averaging done in decision trees. For reasons which will become clear, we call (2) the Markovian rollback formula.

\section{Factoring}

In the model of Figure 1, a background mortality rate $\mu$ is present for both the Well and Post Stroke states. Background mortality is really a process operating in parallel with the stroke process. It can therefore be factored from the stroke model. The result appears in Figure 4. We say that the transient ischemic attack model of Figure 1 is the (Cartesian) product of the Stroke and Background Mortality factors in Figure 4. One advantage of this factoring is that it simplifies the model construction process. The modeler may first deal with issues involving stroke without having to worry about background mortality.

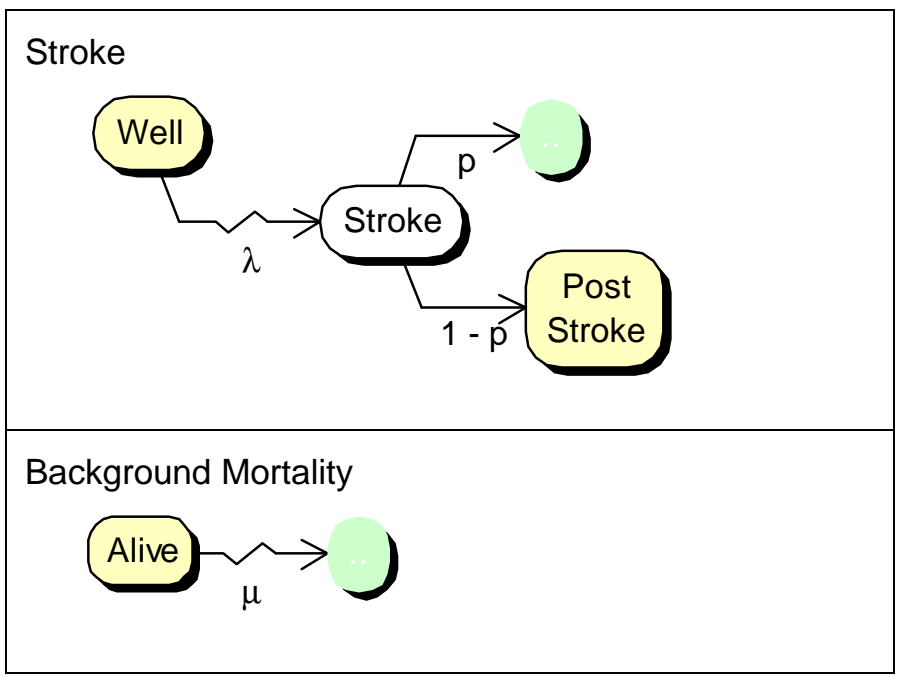

Figure 4: Factoring background mortality out of the stroke model of Figure 1.

Similarly, background mortality can be dealt with independently. For example, the background mortality model in Figure 4 is too simple to be realistic because actual human mortality rates are age dependent, not constant. A more accurate model of human mortality can be constructed by using the Coxian model (Cox 1955, Hazen, Pellissier and Sounderpandian 1998) depicted in Figure 5. The parameters and the number of stages in a Coxian model can be chosen to arbitrarily well approximate human survival durations. The Coxian factor can be substituted 
for simple background mortality in Figure 4, resulting in an improved model. The Cartesian product tree need never be explicitly constructed (but see Hazen, Pellissier and Sounderpandian 1998 for a picture). Hazen (1993) discusses a rollback procedure for factored stochastic trees.

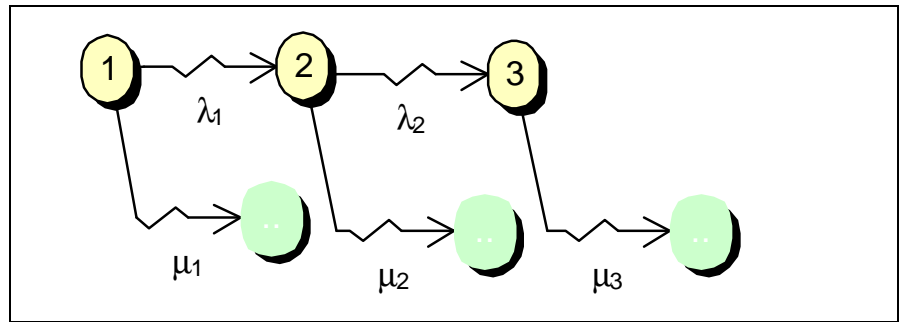

Figure 5: A Coxian mortality factor, in which mortality rate $\mu_{i}$ depends on the stage of the process.

Hazen (1993) constructs a six-factor stochastic tree model of the use of an anticoagulant drug warfarin to treat dilated cardiomyopathy. The model is based on Tsevat et al. (1989). The Systemic Embolism factor for this model appears in Figure 6, and the Anticoagulant Status factor in Figure 7. Three useful modeling features are illustrated: First, stochastic trees can have transition cycles, which allow the possibility of repeated visits to a state. Second, rates or probabilities in one factor can depend on the states of other factors. Finally, transitions in one factor can trigger transitions in other factors.

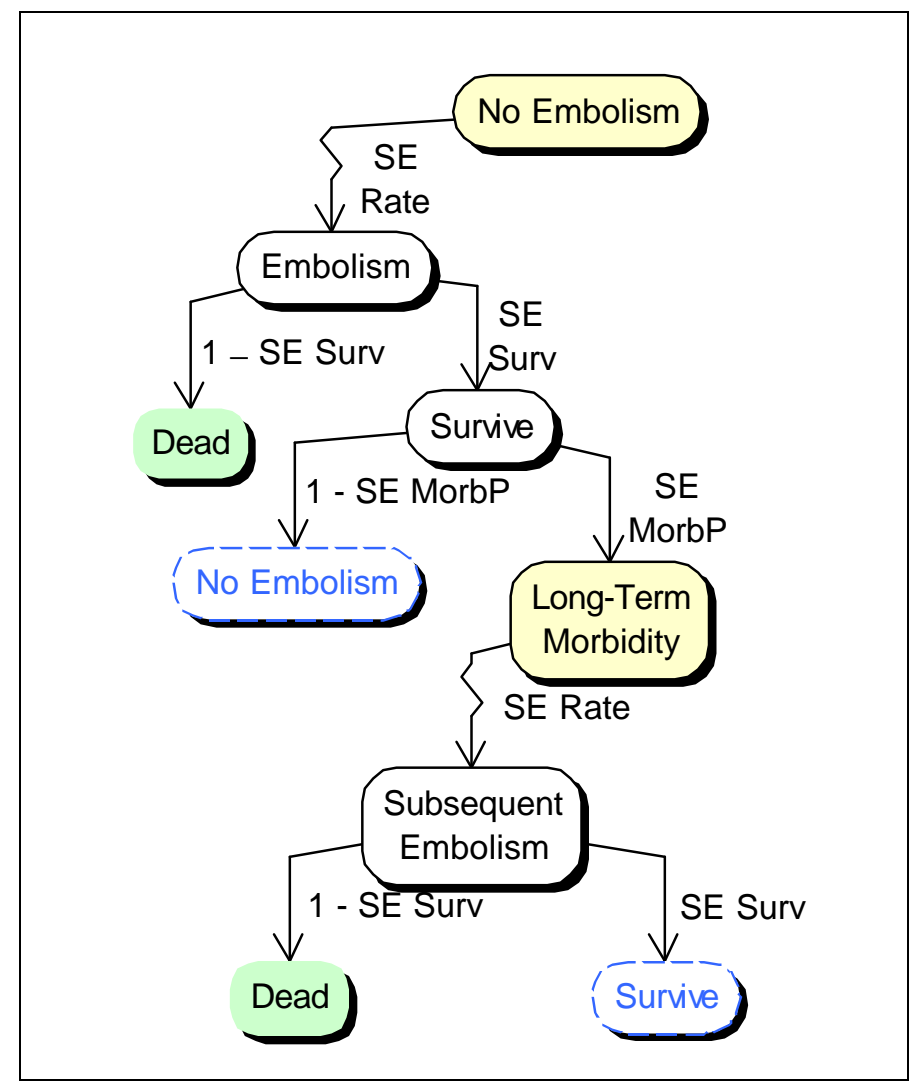

Figure 6: The Systemic Embolism factor from a sixfactor model of the use of warfarin in dilated cardiomyopathy. Nodes with dashed-line borders refer to previous nodes in the tree. For example, from the node Subsequent Embolism transition is possible (with probability SE Surv) to the prior node Survive. So repeated visits to Survive, Long-Term Morbidity and No Embolism are possible. Moreover, the rate of systemic embolism (SE Rate) depends on the state of the Anticoagulant Status factor (Figure 7). 


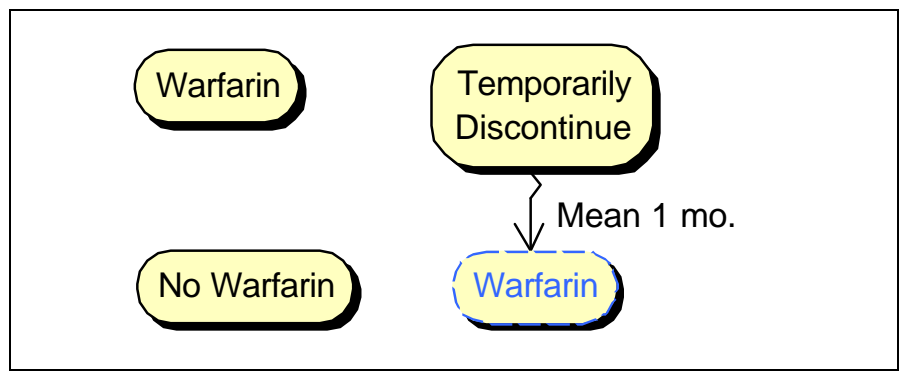

Figure 7: The Anticoagulant Status factor in the warfarin model. An embolism occurrence in the Systemic Embolism factor triggers a transition from No Warfarin to Warfarin in this factor. A hemorrhage in the Systemic Hemorrhage factor (not shown here) triggers a transition here from Warfarin to Temporarily Discontinue.

\section{FACTORING PREFERENCE COMPONENTS FROM A STOCHASTIC TREE}

\section{Updateable-State Preference Summaries}

The Markovian rollback formula (2) for mean quality-adjusted duration presupposes that every state $y$ can be assigned a quality weight $v(y)$ as well as a discount rate $a(y)$. However, sometimes the quality weight at a given state $y$ should depend not only on $y$ but also on the process history prior to entering $y$. For example, in Figure 6 the process begins in the state No Embolism, and may return there after visiting the state Long-Term Morbidity. Because the effects of long-term morbidity are permanent, the quality weight assigned to $N o$ Embolism should depend on whether Long-Term Morbidity has been previously visited. This cannot be accounted for in the Markovian rollback procedure (2).
The dependence of quality weights (and discount rates as well) on the prior history of the process can be incorporated into an efficient rollback procedure by introducing the notion of an updateable-state preference summary (Hazen and Sounderpandian 1999, Hazen and Pellissier 1996). Suppose that the quality weight at state $y$ can depend on the entire sequence $\mathbf{y}=\left(x_{0}, x_{1}, \ldots, x_{k}, y\right)$ of states visited up to and including $y$. Suppose the same is true for the discount rate at $y$. A state preference summary is a function $e(\cdot)$ which assigns a preference summary $r=e(\mathbf{y})$ to every sequence of states $\mathbf{y}$ so that the quality weight and the discount rate at $\mathbf{y}$ depends only on $r=e(\mathbf{y})$. We say that $e(\cdot)$ is has updateable states if there is a function $\theta(q, y)$ such that if $\mathbf{y}=(\mathbf{x}, y)$ and $q=$ $e(\mathbf{x})$, then $e(\mathbf{y})=\theta(q, y)$. In other words, the preference summary at $\mathbf{y}=(\mathbf{x}, y)$ depends only on the last state $y$ and the preference summary at $\mathbf{x}$. When an updateable-state preference summary exists, then rollback operations analogous to (2) may be performed. The notion of preference summary was introduced for discrete-time models by Meyer (1976), who called it state descriptor.

Updateable-state preference summaries are plentiful. If quality weights and discount rates depend only on the current state, as we have been assuming, then $e((\mathbf{x}, y))=y$ is an updateable-state preference summary, which might aptly be called a Markovian preference summary (hence the 
terminology Markovian rollback for (2)). The update function is $\theta(q, y)=y$. Other examples of updateable state summaries are the count of visits to a particular state, whether (Yes or No) a state has ever been visited, and which of a subset of states has been most recently visited. If states are ordered from best to worst in some fashion, then the best state visited so far is an updateable-state summary, but the second-best state so far is not (if there are more than two states).

Hazen and Sounderpandian derive the following rollback equation for mean discounted qualityadjusted duration $L(H \mid q)$ at the generic stochastic fork $H$ in (1) when there is an updateable-state preference summary $q$ prior to $H$ :

$L(H \mid q)=\frac{v(r)+\sum_{i} \lambda_{i} L\left(K_{i} \mid r\right)}{a(r)+\sum_{i} \lambda_{i}}, \quad r=\theta(q, y)$

At the analogous chance fork in which branches are labeled by probabilities $p_{i}$, the rollback formula is

$$
L(H \mid q)=\sum_{i} p_{i} L\left(K_{i} \mid r\right) . \quad r=\theta(q, y)
$$

Note that these equations require the computation of mean discounted quality-adjusted duration $L(H \mid q)$ for every possible preference summary $q$ which could occur prior to the initial node $y$ of $H$.

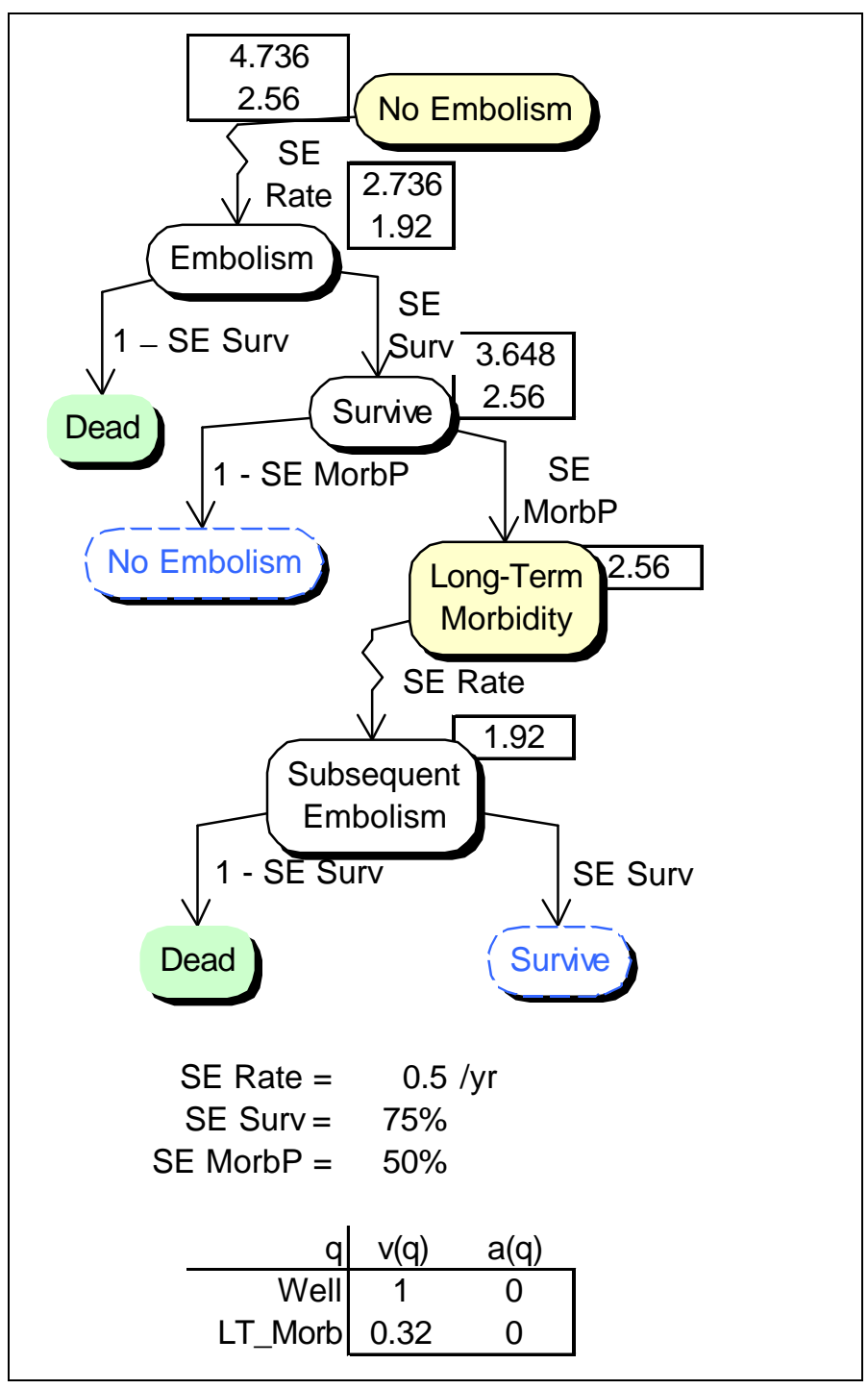

Figure 8: Rollback using the updateable-state preference summary of whether the state Long-Term Morbidity has yet been visited. There are two rollback values at every state except Long-Term Morbidity and Subsequent Embolism, corresponding to the two possible values (Well or LT_Morb) of the preference summary.

Figure 8 shows an example rollback for the Systemic Embolism stochastic tree introduced above. The state preference summary is whether the state Long-Term Morbidity has yet been visited, and consists of the two possibilities \{Well, $\left.L T \_M o r b\right\}$. There are therefore two rollback 
values at each node in Figure 8, except at LongTerm Morbidity and Subsequent Embolism, where the only possible value of the preference summary is $L T \_M o r b$.

\section{Factoring to Recover Markovian Rollback}

One of the main results of this paper is that by suitably factoring a stochastic tree, one may always transform the updateable-state rollback (3) to Markovian rollback (2) on the factored tree. We formally derive this result in the next subsection. Here we illustrate how it may be done for the systemic embolism example of Figure 8.

Generally speaking, the idea is to factor all preference summary information from the stochastic tree. In the systemic embolism example, the preference summary is whether the state LongTerm Morbidity has yet been visited. Therefore, we create a new factor Morbidity Status with possible states Well and Long-Term Morbidity, and we remove Long-Term Morbidity from the original systemic embolism tree. The result is displayed as a two-factor stochastic tree in Figure 9. We have in effect factored out the essential preference information. Note that one of the transitions Survive $\rightarrow$ No Embolism in the new Systemic Embolism factor now triggers transition from Well to LT_Morb in the Morbidity Status factor.
Systemic Embolism

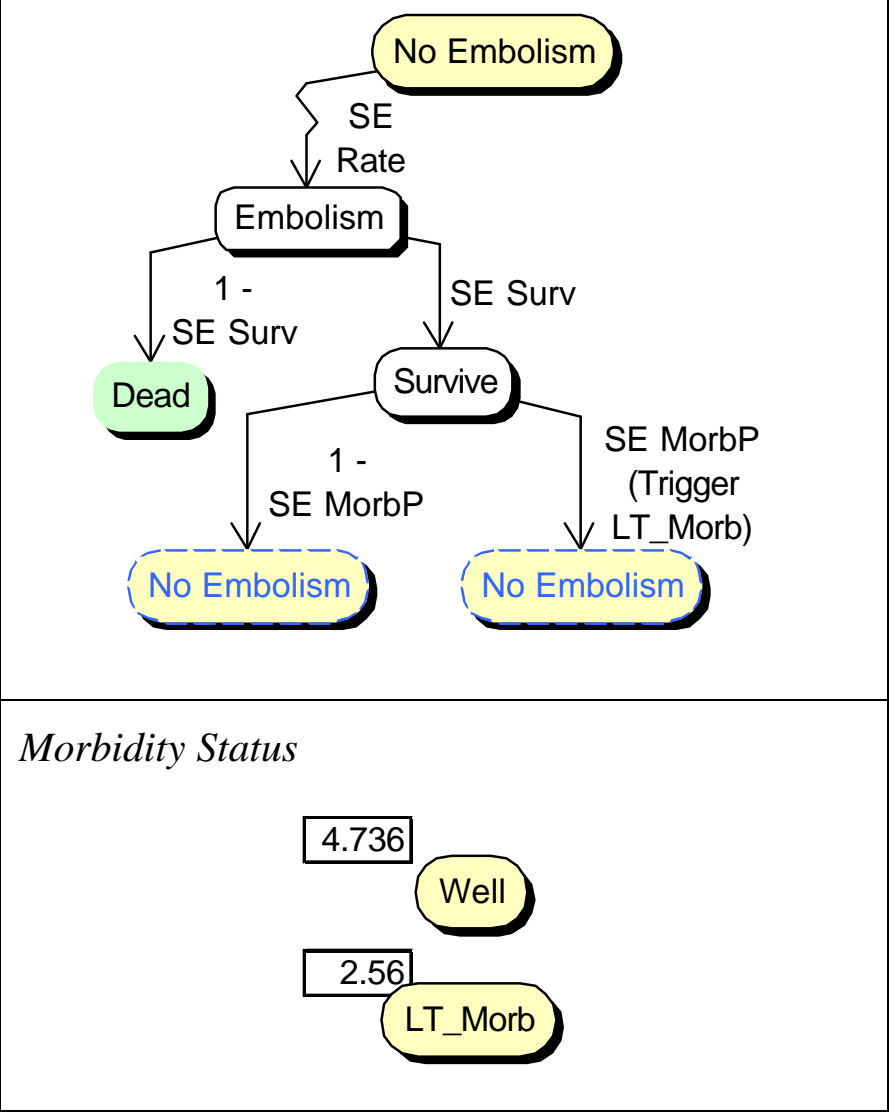

Figure 9: Factoring morbidity status from the Systemic Embolism model of Figure 8. Transition from Well to LT_Morb in the Morbidity Status factor can occur only when triggered by the transition Survive $\rightarrow$ No Embolism in the Systemic Embolism factor. Mean quality-adjusted life years for the product of these two factors are shown next to the appropriate states. These quantities were calculated using the Markovian rollback formulas and are identical to the results in Figure 8 obtained using updateable-state rollback formulas.

Recall that the overall stochastic tree is now the Cartesian product of the Systemic Embolism factor and the Morbidity Status factor in Figure 9, so has states of the form $(y, q)$, where $q$ is morbidity status. Therefore what was previously a preference summary $q$ in the original model of 
Figure 8 has become part of the state information in the factored model of Figure 9. For this reason, the preference summary for the factored model is Markovian, and the Markovian rollback formulas can be employed. The numerical results of Markovian rollback are displayed in Figure 9, and are of course identical to the values in Figure 8. Computationally, the Markovian rollback is identical to the previous updateable-state rollback. However, the factored form of the model in Figure 9 gives much greater insight into model structure: The Systemic Embolism factor in Figure 9 succinctly summaries probabilistic structure, and the Morbidity Status factor summaries preference structure.

Compared to the simple Markovian preference summary, updateable-state preference summaries appear to allow much greater flexibility in preference representation. However, this appearance is illusory because as the same flexibility can be acquired by including the preference summary $q$ in the state of the stochastic tree and using a Markovian summary.

At first sight, this result appears to diminish the significance of updateable-state utility. From a computational standpoint one need never implement the updateable-state rollback equations (3) and (4) because Markovian rollback (2) over augmented-state stochastic trees will do. However, from the viewpoint of model construction and presentation, it is still advantageous to think in terms of updateable-state preference summaries because these may be factored from the model in a way that eases model construction and simplifies model presentation.

As promised, the preference factoring methodology discussed here by example is presented in a general setting in the following section. Readers not interested in the mathematical details can skip the next section without loss of continuity.

\section{General Results on Preference Factoring}

The Markovian rollback formulas (2) can be generalized to allow for tolls and discount multipliers, as I now explain. Let $E U(H \mid x)$ be the expected Markovian utility of the stochastic tree $H$ in (1) given that $y$ is reached from preceding state $x$. From Hazen and Pellissier (1996), the rollback formulas (2) generalize to

$$
\begin{aligned}
& E U(H \mid x)=w(y \mid x)+\Delta w(y \mid x) E U_{0}(H) \\
& E U_{0}(H)=\frac{v(y)+\sum_{i} \lambda_{i} E U\left(K_{i}\right)}{a(y)+\sum_{i} \lambda_{i}}
\end{aligned}
$$

Here $w(y \mid x)$ is the negative of the toll from $x$ to $y$, and $\Delta w(y \mid x)$ is the discount multiplier from $x$ to $y$.

Updateable-state utility can be generalized to allow for tolls and discount multipliers as well. From Hazen and Sounderpandian (1999), one may 
derive the following generalization of the updateable-state rollback formulas (3):

$$
\begin{aligned}
& E u(H \mid q)=w(y \mid q)+\Delta w(y \mid q) E u_{0}(H \mid q) \\
& E u_{0}(H \mid q) \\
& =\frac{v(r)+\sum_{i} \lambda_{i} E u\left(K_{i} \mid r\right)}{a(r)+\sum_{i} \lambda_{i}}, \quad r=\theta(q, y)
\end{aligned}
$$

Again, $w(y \mid q)$ and $\Delta w(y \mid q)$ are the negative toll and the discount factor respectively at $y$ given preference summary $q$. Both the Markovian rollback formulas (5) and the updateable-state rollback formulas (6) are true expected utility formulas, that is, each results from taking the expectation of a utility function defined over sample paths.

Consider the situation described in the systemic embolism example of the previous section, in which one or more states are present not for any stochastic reason but only for their impact on preference. We formalize this notion as follows. Given a stochastic tree $H$ with states in $Y$, we say that an equivalence relation $\equiv$ over $Y$ is a stochastic equivalence relation if whenever $x \equiv x^{\prime}$, then there is a one-to-one correspondence between the arcs immediately succeeding $x$ and the arcs immediately succeeding $x^{\prime}$ such that

(a) corresponding arcs have the same attached rate (or probability). (b) if $y_{i}$ is a node successor of $x$ and $y_{i}^{\prime}$ is the corresponding node successor of $x^{\prime}$ then $y_{i} \equiv y_{i}^{\prime}$.

Note that if $x \equiv x^{\prime}$, then starting the process in state $x$ is stochastically identical to starting the process in state $x^{\prime}$.

Let $x^{\equiv}$ be the set of all nodes $x^{\prime}$ stochastically equivalent to $x$. By merging all stochastically equivalent states, and identifying the corresponding successor arcs, one can form the stochastic tree $H^{\equiv}$ (sometimes denoted $H / \equiv$ ), whose states and arcs are equivalence classes modulo $\equiv$ of states and arcs in $H$.

For example, in the systemic embolism tree $H$ of Figure 6, notice that

$$
\text { No Embolism } \equiv \text { Long-Term Morbidity }
$$

Subsequent Embolism $\equiv$ Embolism.

When these equivalent states and their corresponding arcs are merged, the result is the Systemic Embolism factor $H^{\equiv}$ of Figure 9.

If one's only goal is to display probabilistic structure or to compute probabilities, then it is better to use $H^{\equiv}$ because it has fewer nodes and arcs. However, if one wishes to compute expected utilities under an updateable-state preference structure, then one must revert to $H$, because stochastically equivalent states need not have the same preference summaries. For instance in the 
Systemic Embolism model $H$, the state Embolism has possible preference summaries $\{$ Well, $\left.L T \_M o r b\right\}$ but the stochastically equivalent state Subsequent Embolism has only LT_Morb as a possible preference summary.

Our main result of this section follows. We show that the method of the previous section works in general; that is one may reduce an updateable-state expected utility computation to a Markovian expected utility computation by factoring out preference summaries and identifying stochastically equivalent states. Here are the details.

Construct a product tree $H^{\equiv} \otimes Q$ as follows: Let $H^{\bar{E}} \otimes Q$ have states $\left(x^{\equiv}, q\right)$, where $q$ is a possible preference summary up to and including some $x^{\prime} \in x^{\equiv}$. For every $\operatorname{arc} x^{\equiv} \rightarrow y^{\equiv}$ in $H^{\overline{ }}$, every $x^{\prime}$ $\in x^{\equiv}$, and every preference summary $q$ which can occur at $x^{\prime}$, add in $H^{\equiv} \otimes Q$ the $\operatorname{arc}\left(x^{\equiv}, q\right) \rightarrow\left(y^{\equiv}, r\right)$ where $r=\theta\left(q, x^{\prime}\right)$. This product tree can be visualized in factored form: The transition $x^{\equiv} \rightarrow y^{\equiv}$ in $H^{\bar{\Xi}}$, triggers the transition $q \rightarrow r$ in $Q$.

Suppose $w(y \mid q), \Delta w(y \mid q), v(q)$ and $a(q)$ are the component functions of the original updateablestate utility function $u(\cdot)$. We make the assumption that tolls and discount factors depend only on preference summaries, that is, $w(y \mid q)$ and $\Delta w(y \mid q)$ are functions only of $q$ and $r=\theta(q, y)$. For convenience we write

$$
w(y \mid q)=w(r \mid q)
$$

$$
\Delta w(y \mid q)=\Delta w(r \mid q)
$$

Define the components of a Markovian utility function $U(\cdot)$ over product trees $H^{\equiv} \otimes Q$ as follows.

$$
\begin{aligned}
& w\left(\left(y^{\equiv}, r\right) \mid\left(x^{\equiv}, q\right)\right)=w(r \mid q) \\
& \Delta w\left(\left(y^{\equiv}, r\right) \mid\left(x^{\equiv}, q\right)\right)=\Delta w(r \mid q) \\
& v\left(y^{\equiv}, r\right)=v(r) \\
& a\left(y^{\equiv}, r\right)=a(r) .
\end{aligned}
$$

We have the following result.

Theorem 1: Suppose preference over stochastic trees having possible states $y \in Y$ is represented by an updateable-state utility function $u(\cdot)$ whose tolls and discount factors depend only on preference summaries. Let $H$ be an arbitrary stochastic tree with states in $Y$, and let $\equiv$ be a stochastic equivalence relation over $H$. Suppose the stochastic tree $H^{\equiv} \otimes Q$ and Markovian utility $U(\cdot)$ are constructed as described above. For any $\left(y^{\equiv}, r\right)$ in $H^{\equiv} \otimes Q$, let $H\left(y^{\equiv}, r\right)$ be the subtree with initial state $\left(y^{\equiv}, r\right)$, and let $H(y)$ be the subtree of $H$ with initial state $y$. Then

$$
E U\left(H\left(y^{\equiv}, r\right) \mid\left(x^{\equiv}, q\right)\right)=E u(H(y) \mid q)
$$




\section{Arthritis Modeling with Updateable-State} Utility

My colleagues and I (Chang, Pellissier and Hazen 1996) examined the cost-effectiveness of total hip replacement surgery (total hip arthroplasty, or THA). An estimated 120,000 hip replacements are performed per year in North America (Harris and Sledge 1990). THA is an elective, high cost procedure which reduces disability but does not extend life, and which has less expensive short-term alternatives. It is therefore particularly vulnerable to questions of cost-effectiveness. Nevertheless, we concluded that when quality of life is taken into account, THA is one of the most cost-effective of medical procedures, comparable or superior to wellaccepted procedures such as cardiac bypass or renal dialysis.

Our cost-effectiveness analysis was performed using a stochastic tree model of hip surgery and its consequences. Our model at that time did not exploit updateable-state preference summaries. However, it is easy to recast our efforts in those terms, and the resulting formulation and presentation given below, while equivalent, gives considerably greater insight than the original. A summary of the model as originally conceived may be found in Hazen, Pellissier and Sounderpandian (1998).
We chose to characterize the effectiveness of THA in terms of functional outcome measured by the four-state American College of Rheumatology (ACR) functional status classification. The four classes on the ACR scale are as follows:

I Complete ability to carry on all usual duties without handicaps.

II Adequate for normal activities despite handicap of discomfort or limited motion in one or more joints.

III Limited only to little or none of duties of usual occupation or self-care.

IV Incapacitated, largely or wholly bedridden or confined to wheelchair; little or no self care.

A candidate for THA is typically in functional class III. The result of the THA procedure is usually an immediate transition to functional class I or II. However, short- or long-term complications may arise. The presence of infection might cause the prosthesis to fail over time (septic failure). The prosthesis might fail for a variety of other reasons such as mechanical loosening, prosthesis breakage, or dislocation (aseptic failure). Should any of these failures occur, revision surgery is required which is often less successful than the initial THA. A septic failure can cause permanent degradation in functional status. Small mortality rates accompany both initial THA and revision surgeries. 
How can we construct an updateable-state preference summary for this problem? Clearly the ACR functional status measure should somehow be involved. We made several relevant assumptions early in the modeling effort. First, we assumed that all revision surgeries which the patient survives would either succeed or fail. Therefore, surviving patients would have either successfully addressed or unsuccessfully addressed prosthesis failures. We secondly assumed that in the absence of septic history, a successfully addressed aseptic failure would place the patient into functional class II, whereas an unsuccessfully addressed aseptic failure would result in functional class III. In the presence of septic history, the respective outcomes would be classes III and IV. Finally, a successfully addressed septic failure would place the patient into functional class III, and an unsuccessfully addressed septic failure would result in functional class IV. Patients for whom revision fails may undergo further revisions, but due to limitations in available bone stock, we decided it was not realistic to allow in the model more than three revision surgeries.

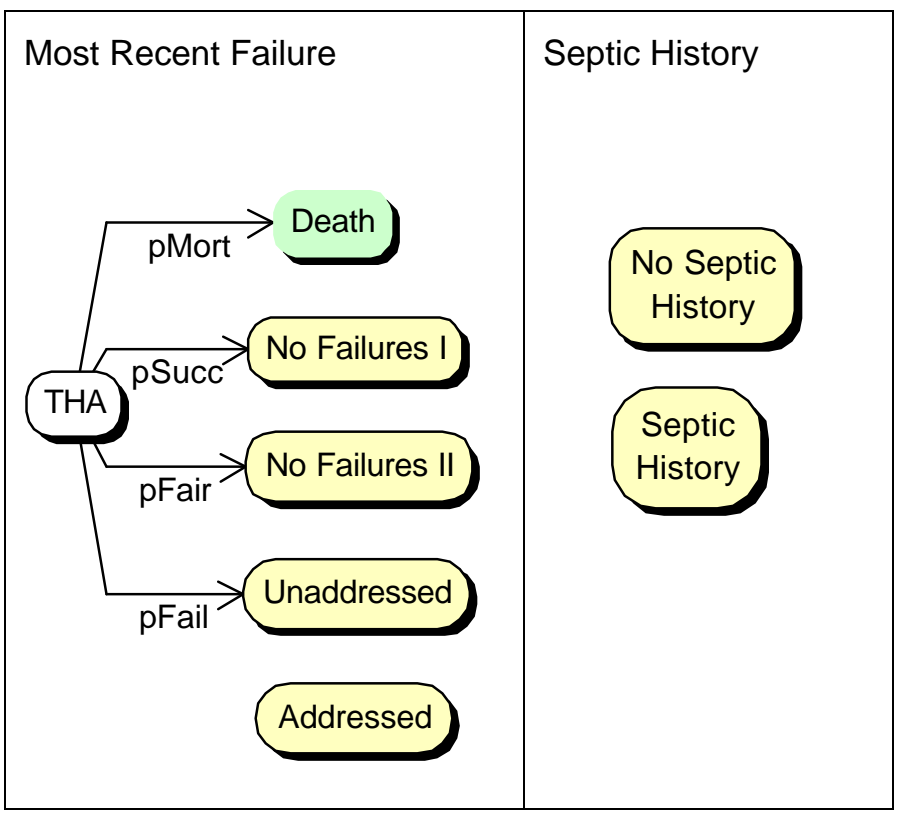

Figure 10: Factors of the THA model corresponding to the bivariant preference summary (Failure History, Septic History). The initial state of the Most Recent Failure factor is determined by the outcome of initial THA, as indicated, whereas the initial state of the Septic History factor is No_Septic_History. Overall utility in the THA model is a direct function of time spent in the combined states of these two factors.

Based on these comments, it is apparent that functional status depends on a bivariate preference summary

$$
q=(\text { Most Recent Failure, Septic History }) .
$$

Summary states are listed in Figure 10, which depicts the two stochastic factors arising from this updateable-state preference summary. The exact dependence of functional status on Failure History and Septic History is given in Table 1. 


\begin{tabular}{|ll|c|}
\hline $\begin{array}{l}\text { Failure } \\
\text { History }\end{array}$ & Septic History & $\begin{array}{c}\text { Functional } \\
\text { Status }\end{array}$ \\
\hline No Failures I & - & I \\
No Failures II & - & II \\
All Failures & No Septic History & II \\
Addressed & & \\
Unaddressed & No Septic History & III \\
Failures & & \\
& Septic History & IV \\
\hline
\end{tabular}

Table 1: Functional status depends on Failure History and Septic History.

We can now assign quality factors $v(q)$ for Markovian utility by assessing patients' quality factors for functional classes I, II, III, IV. Based on empirical work by ourselves and others, we assigned the approximate values indicated in Table 2. Of course these values will differ across individuals.

\begin{tabular}{|l|llll|}
\hline Functional Status & I & II & III & IV \\
\hline Quality factor & 1.0 & 0.8 & 0.5 & 0.3 \\
\hline
\end{tabular}

Table 2: The quality factor for Markovian utility depends on functional status. These are approximate values based on empirical work by ourselves and others.

The primary stochastic factor for the THA model is shown in Figure 11. Transitions in this factor affect utility by triggering transitions in the Failure History and Septic History factors.
Other factors were required in the model as well. A revision count factor was necessary to limit the number of revisions to three. Factors which count aseptic revisions and septic revisions separately and a factor which counts the most recent revision type (aseptic or septic) were needed because subsequent failure rates and revision success probabilities depend on these. Finally, the model includes an age- and gender-specific Coxian mortality factor.

The results of rollback for this stochastic tree model are shown in Table 3 for two individuals of differing gender and age. For both, THA roughly doubles discounted expected remaining qualityadjusted lifetime.

The purpose of preference factoring is to explicitly present features of the model on which overall utility most directly depends. As we hope this example illustrates, an analyst who employs this technique can clarify preference modeling assumptions and open the model to inspection and critique by others. 


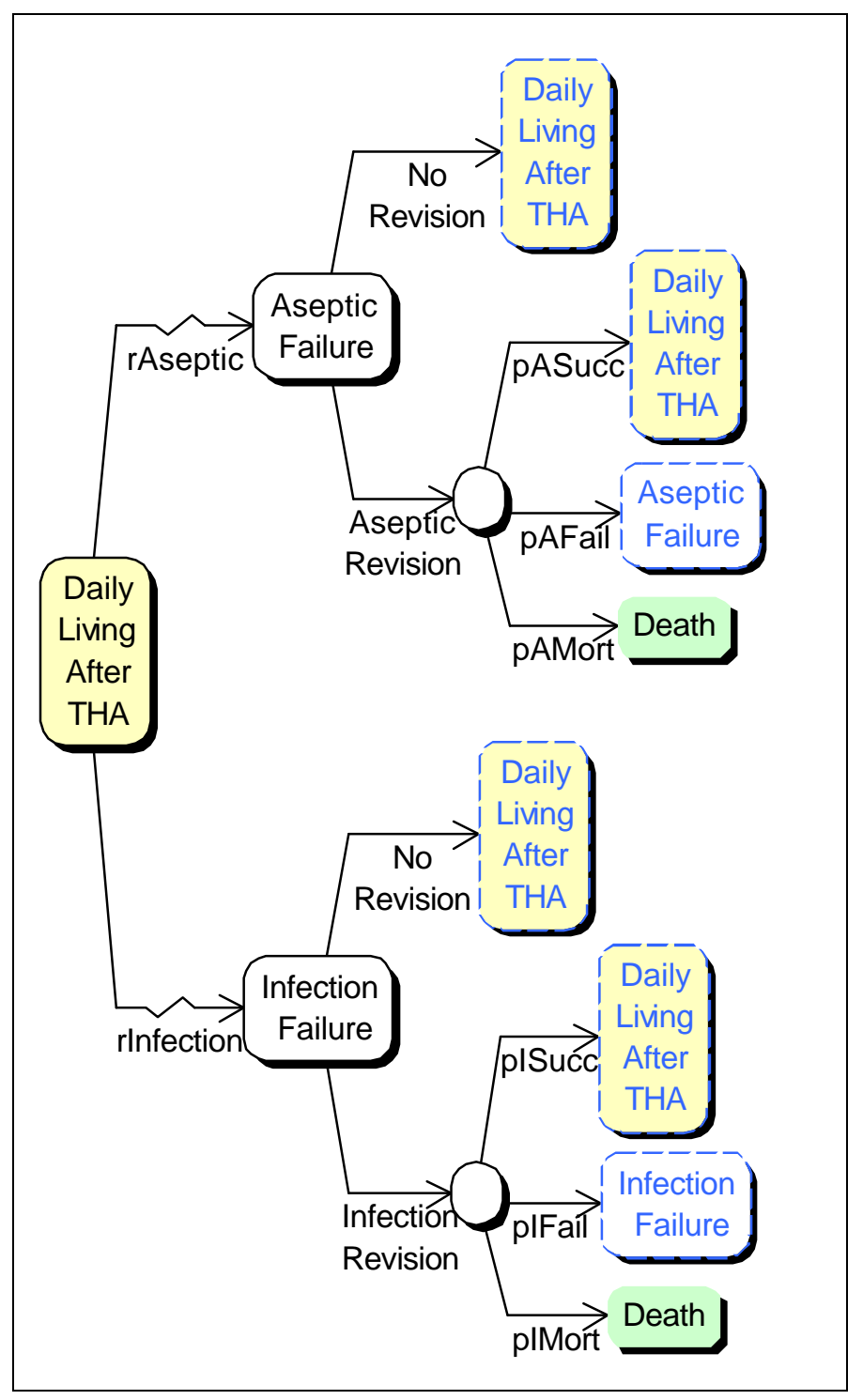

Figure 11: The primary stochastic factor for the THA model. Transition to Infection_Failure triggers a transition to Unaddressed in the Most Recent Failure factor, and a transition to Septic_History in the Septic History factor. Infection revision is performed if the total revision count (factor not shown) is less than three. Subsequent successful revision triggers a transition to Addressed in the Most Recent Failure factor. Similar triggers are invoked upon transition to Aseptic_Failure. All rates and probabilities in this factor depend on other factors not shown (Aseptic Revision Count, Infection Revision Count, Last Revision Type).

\begin{tabular}{|l|c|c|}
\hline \multirow{2}{*}{} & \multicolumn{2}{|c|}{ Discounted quality-adjusted years } \\
\cline { 2 - 3 } & THA & No THA \\
\hline $\begin{array}{l}\text { White female } \\
\text { age 60 }\end{array}$ & 13.70 & 6.82 \\
$\begin{array}{l}\text { White male } \\
\text { age 85 }\end{array}$ & 4.16 & 2.16 \\
\hline
\end{tabular}

Table 3: Comparison of THA versus No THA for two different individuals. An annual discount rate of 5\% was used.

\section{MULTIATTRIBUTE DECOMPOSITION OF MARKOVIAN UTILITY}

In this section we examine utility decompositions which can arise when factors in a stochastic tree are treated as attributes in a multiattribute utility function. We consider only Markovian utility functions, noting that the results have implications for updateable-state utility as well because of the connection established by Theorem 1.

We consider the case of two-factor stochastic trees, since the multi-factor case follows naturally. We denote states in the first factor by $y, y^{\prime}, y_{0}, y_{1}$ and so on; states in the second factor by $z, z^{\prime}, z_{0}, z_{1}$ and so on; and states in the product tree by $x=$ $(y, z), x^{\prime}=\left(y^{\prime}, z^{\prime}\right)$, etc. Let $y^{*}$ and $z^{*}$ be distinguished states (e.g., Well) and for Markovian utility. We assume $v\left(y^{*}, z^{*}\right)>0$.

We use the following graphical notation. The display 


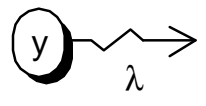

denotes the occupation of state $y$ subject to

mortality rate $\lambda$. For utility assessment purposes, it is often convenient to let $\lambda$ be time dependent, so this display denotes a duration $T$ sojourn in $y$ followed by process termination (death, in the medical context), where $T$ is a continuous-valued nonnegative random variable with hazard rate function $\lambda(t)$. The display

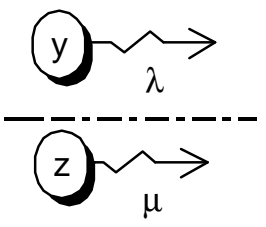

denotes a two-factor scenario in which $y$ is occupied with mortality rate $\lambda$ in the first factor and $z$ is occupied with mortality rate $\mu$ in the second factor. Because termination in either factor forces termination of the entire process, this is equivalent to the product tree

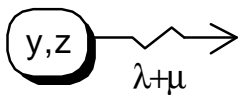

\section{The risk-neutral preference interpretation for Markovian utility}

Given a hazard rate function $\lambda(t)$, let $m(\lambda)$ denote the mean of the corresponding random variable. The following elementary results are useful.
Proposition 1: If $T$ has hazard rate function $\lambda(t)$ then

$$
\begin{aligned}
& \text { (i) } E\left[\int_{0}^{T} e^{-a t} d t\right]=m(\lambda+a) \\
& \text { (ii) } E\left[e^{-a T}\right]=1-a \cdot m(\lambda+a)
\end{aligned}
$$

Markovian utility over sample paths can be expressed recursively. Let $x^{t} h$ be a sample path beginning with a duration- $t$ sojourn in state $y$ and followed by some other sample path $h$. If $U\left(x^{t} h \mid x^{\prime}\right)$ is the Markovian utility of sample path $x^{t} h$ given preceding state $x^{\prime}$, then

$$
U\left(x^{t} h \mid x^{\prime}\right)=w\left(x \mid x^{\prime}\right)+\Delta w\left(x \mid x^{\prime}\right) U_{0}\left(x^{t} h\right)
$$

$$
U_{0}\left(x^{t} h\right)=\int_{0}^{t} v(x) e^{-a(x) s} d s+e^{-a(\mathrm{x}) t} U(h \mid x)
$$

(Hazen and Pellissier 1996). As previously mentioned, $v(x)$ is a quality weight at state $x, a(x)$ is a state-dependent discount rate, $w\left(x \mid x^{\prime}\right)$ is the negative of a toll in the transition from $x^{\prime}$ to $x$, and $\Delta w\left(x \mid x^{\prime}\right)$ is a discount multiplier for that transition.

Denote by $x^{\lambda}$ a duration- $T$ sojourn in state $x$, where $T$ has hazard rate function $\lambda$. Note that for Markovian utility without toll or discount multiplier, we have

$$
u\left(x^{\lambda}\right)=E\left[v(x) \int_{0}^{T} e^{-a(x) t} d t\right]=v(x) m(\lambda+a(x))
$$

For constant hazard rate $\lambda$, the result is 


$$
u\left(x^{\lambda}\right)=\frac{v(x)}{\lambda+a(x)} .
$$

One interpretation of this equation is that under Markovian utility, a risk-sensitive sojourn with hazard rate $\lambda$ in state $x$ is equivalent to a riskneutral sojourn with hazard rate $\lambda+a(x)$ in state $\mathrm{x}$. We call this the risk-neutral interpretation of Markovian utility.

A strategy we have used (Hazen, Hopp and Pellissier 1991) to assess the risk-attitude parameter of Markovian utility is to elicit from subjects an immediate mortality probability $p$ such that the subject is indifferent between the current mortality rate and a chance $p$ of surviving immediate death with an improved mortality rate, that is,

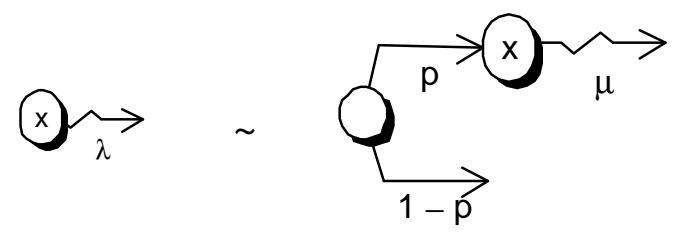

Then equating expected utilities yields

$$
v(x) m(\lambda+a(x))=p v(x) m(\mu+a(x)) .
$$

This equation can be solved for $a(x)$ in most situations (e.g., when $\mu=\lambda+c$ for some nonnegative constant $c$ ).

\section{The failure of full utility independence}

In any multiattribute context, it is natural to attempt to invoke utility independence. The naïve extension of utility independence from attributes to stochastic factors would run as follows: Call factor A utility independent of factor $B$ if preference for stochastic trees in factor A do not depend on the particular stochastic tree in factor B. This definition must be amended to include the requirement that there are no parameter dependencies or triggers linking the two factors ${ }^{\dagger}$. However, it is still not a useful definition because despite appearances to the contrary, transition rates do not necessarily attach to particular factors. For example, due to the way the Cartesian product of stochastic trees is defined, we have the equalities

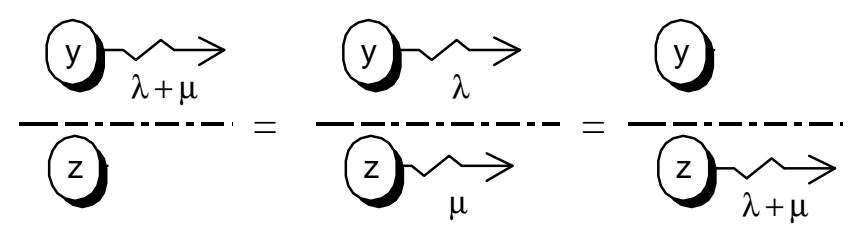

holding because all three are equal to the product tree

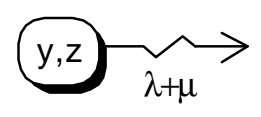

Utility independence of factor 1 from factor 2 would imply, for example, that the indifference

\footnotetext{
${ }^{\dagger}$ This is an extension of a less familiar but equivalent form of utility independence: Attribute $X$ is utility independent of attribute $\mathrm{Y}$ if for any lotteries $x, x^{\prime}, y, y^{\prime}$ with $x, x^{\prime}$ stochastically independent of $y, y^{\prime}$ we have $(x, y)$ preferred to $\left(x^{\prime}, y\right)$ if and only if $\left(x, y^{\prime}\right)$ preferred to $\left(x^{\prime}, y^{\prime}\right)$.
} 


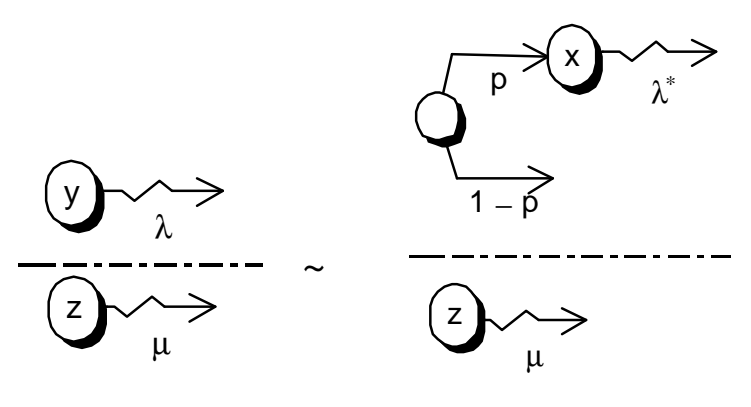

if holding for one $z, \mu$ combination, must hold for all. In product tree terms, this indifference translates to

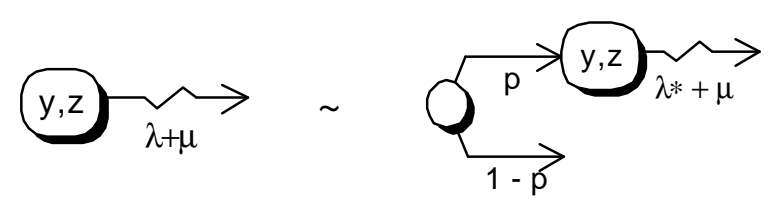

The requirement that this indifference holds for all $\mu$ if it holds for one is almost always false. For Markovian utility and constant mortality rates, the resulting expected utility equation is

$$
\frac{1}{a(y, z)+\lambda+\mu}=\frac{p}{a(y, z)+\lambda^{*}+\mu} .
$$

If this equation holds for one value of $\mu$, it can hold for no others. Therefore full utility independence cannot hold.

\section{Non-interfering utility independence}

As a less restrictive assumption, it might be reasonable to require that one factor be utility independent of another factor as long as the other factor never changes state, and therefore never interferes with the first factor. Call this non- interfering utility independence. It would imply, for example, that the indifference

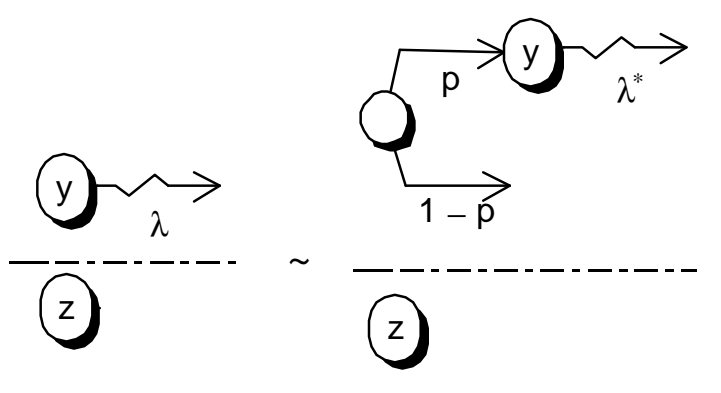

if holding for one nonfatal $z$, would hold for all nonfatal $z$. In product form, this indifference is

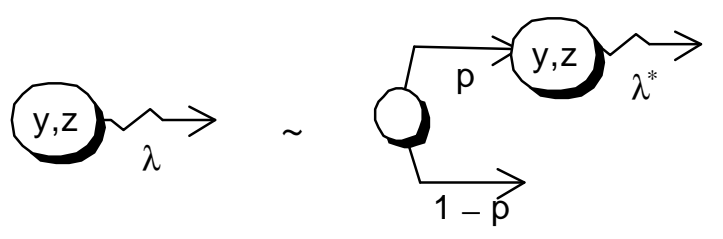

Since this indifference determines the risk attitude parameter $a(y, z)$, it would follow that $a(y, z)$ is independent of $z$. If each factor is non-interfering utility independent of the other, then $a(y, z)$ can depend on neither $y$ nor $z$, so is a constant $a(y, z)=$ $a^{*}$. This establishes the first part of the following result.

Theorem 2: Under Markovian utility each factor is non-interfering utility independent of the other if and only if there exist constants $v^{*}>0$ and $a^{*}$, marginal quality-rate functions $v_{1}(y), v_{2}(y)$, marginal tolls $w_{1}\left(y \mid y_{0}\right), w_{2}\left(z \mid z_{0}\right)$, and marginal discount multipliers $\Delta w_{1}\left(y \mid y_{0}\right)>0, \Delta w_{2}\left(z \mid z_{0}\right)>0$ such that 
(i) $a(y, z)=a^{*}$ independent of $y, z$;

(ii) $\frac{v(y, z)}{v^{*}}=\frac{v_{1}(y)}{v^{*}} \frac{v_{2}(z)}{v^{*}}$

where $v^{*}=v\left(y^{*}, z^{*}\right)=v_{1}\left(y^{*}\right)=v_{2}\left(z^{*}\right)$

(iii) $w\left(y, z \mid y_{0}, z\right)=w_{1}\left(y \mid y_{0}\right) \cdot v_{2}(z) \cdot v^{*}$

$$
w\left(y, z \mid y, z_{0}\right)=v_{l}(y) \cdot w_{2}\left(z \mid z_{0}\right) \cdot v^{*}
$$

(iv) $\Delta w\left(y, z \mid y_{0}, z\right)=\Delta w_{l}\left(y \mid y_{0}\right)$

$$
\Delta w\left(y, z \mid y, z_{0}\right)=\Delta w_{2}\left(z \mid z_{0}\right) .
$$

The proof is given in the appendix. Usually one would rescale $v$ so that $v^{*}=1$.

The fact that non-interfering utility independence forces risk attitude $a(y, z)$ to be a constant independent of $y, z$ is disappointing because it reduces the flexibility of the multiattribute Markovian utility model. We have, for example, observed that subjects engaged in immediate mortality scenarios of the type described above have risk attitudes which can depend on health state (Pellissier and Hazen 1994). However, we are aware of no realistic Markovian utility decomposition which allows non-constant $a(y, z)$.

\section{CONCLUSIONS}

We have shown that the useful notion of factoring a stochastic tree model may be extended to the preference domain as well, that is, one may represent updateable-state preference components as one or more factors in a multi-factor decomposition of the stochastic tree. Then one may compute expected updateable-state utility by rolling back an equivalent Markovian utility function over the product tree. The Markovian rollback is computationally equivalent to an updateable-state rollback without preference factoring. However, the explicit factoring of preference components from the stochastic tree eases model formulation and presentation, and resulting transparent form opens the model to inspection and critique by others.

We have also explored multiattribute utility decompositions for Markovian utility. The usual notion of utility independence fails for Markovian utility over stochastic trees. However, the restricted notion of non-interfering utility independence leads to a useful utility decomposition.

\section{REFERENCES}

R.W. Chang, J.M. Pellissier and G.B. Hazen, "A Cost-Effectiveness Analysis of Total Hip Arthroplasty for Osteoarthritis of the Hip", Journal of the American Medical Association 275 (1996) 858-865.

Cox, D.R., "A use of complex probabilities in the theory of stochastic processes," Proceedings of the Cambridge Philosophical Society, 51 (1955) 313-319.

C.A. Gottlob, J.M. Pelliesier, R.L. Wixson, S.H. Stern, S.D. Stulberg, R.W. Chang, "The 
Long-Term Cost-Effectiveness of Total Knee Arthroplasty for Osteoarthritis", Multipurpose Arthritis Center, Northwestern University, Chicago, Illinois.

W.H. Harris and C.B. Sledge, "Total Hip and Total Knee Replacement," New England Journal of Medicine 323 (1990) 725-731.

G.B. Hazen, "Stochastic Trees: A New Technique for Temporal Medical Decision Modeling," Medical Decision Making 12 (1992) 163-178.

G.B. Hazen, "Factored Stochastic Trees: A Tool for Solving Complex Temporal Medical Decision Models," Medical Decision Making. 13 (1993), 227-236.

G.B. Hazen, W.J. Hopp and J.M. Pellissier, "Continuous-Risk Utility Assessment in Medical Decision Making," Medical Decision Making 11 (1991) 294-304.

G.B. Hazen, M. Morrow and E.R. Venta, "A Decision Analysis for the Treatment of Ductal Carcinoma in Situ", in progress, IEMS Department, Northwestern University, Evanston Illinois.

G.B. Hazen and J.M. Pellissier, "Recursive Utility for Stochastic Trees", Operations Research 44 (1996) 788-809.

G.B. Hazen and J. Sounderpandian, "StateTrajectory Preference Summaries for Stochastic Tree Rollback", forthcoming in the FUR 8 proceedings, M. Machina and B. Munier (eds.), Kluwer 1999.

G.B. Hazen, J.M. Pellissier and J. Sounderpandian (1998), "Stochastic tree models in medical decision making", Interfaces 48, 64-80.

Keeney, R.L. and H. Raiffa.. Decisions with Multiple Objectives: Preferences and Value Tradeoffs. Wiley, New York, 1976.
D.B. Matchar and S.G. Pauker, "Transient Ischemic Attacks in a Man with Coronary Artery Disease: Two Strategies Neck and Neck", Medical Decision Making 6 (1986) 239-249.

R.F. Meyer, "Preferences over time", in Keeney and Raiffa (1976), Chapter 9.

J.M. Pellissier, G.B. Hazen and R.W. Chang, "A Continuous-Risk Decision Analysis of Total Hip Replacement", Journal of the Operations Research Society 47 (1996) 776-793.

J.M. Pellissier and G.B. Hazen, "Implementation of Continuous-Risk Utility Assessment: The Total Hip Replacement Decision", SocioEconomic Planning Sciences 28 (1994) 251276.

J. Tsevat, M.H. Eckman, R.A. McNutt, S.G. Pauker, "Warfarin for Dilated Cardiomyopathy: A Bloody Tough Pill to Swallow?", Medical Decision Making 9 (1989) 162-169. 


\section{APPENDIX: PROOFS OF THEOREMS}

\section{Proof of Theorem 1}

Let $H\left(y^{\equiv}, r\right)$ be a stochastic fork at state $\left(y^{\equiv}, r\right)$ in $H^{\bar{\Xi}}$

$\otimes Q$. Suppose $\left(y^{\equiv}, r\right)$ has successors $\left(z_{i}{ }^{\equiv}, s_{i}\right)$, where $s_{i}=\theta\left(r, z_{i}\right)$. For the Markovian utility $U(\cdot)$ defined in the theorem, the rollback equations (5) become

$$
\begin{gathered}
E U\left(H\left(y^{\equiv}, r\right) \mid\left(x^{\equiv}, q\right)\right)=w\left(\left(y^{\equiv}, r\right) \mid\left(x^{\equiv}, q\right)\right) \\
+\Delta w\left(\left(y^{\equiv}, r\right) \mid\left(x^{\equiv}, q\right)\right) E U_{0}\left(H\left(y^{\equiv}, r\right)\right) \\
E U_{0}\left(H\left(y^{\equiv}, r\right)\right)= \\
=\frac{v\left(y^{\equiv}, r\right)+\sum_{i} \lambda_{i} E U\left(H\left(z_{i}, s_{i}\right)\left(y^{\equiv}, r\right)\right)}{a\left(y^{\equiv}, r\right)+\sum_{i} \lambda_{i}}
\end{gathered}
$$

Apply the definitions (8) to obtain

$$
\begin{aligned}
& E U\left(H\left(y^{\equiv}, r\right)\left(\left(x^{\equiv}, q\right)\right)=w(r \mid q)\right. \\
& +\Delta w(r \mid q) \frac{v(r)+\sum_{i} \lambda_{i} E U\left(H\left(z_{i} \equiv, s_{i}\right)\left(y^{\equiv}, r\right)\right)}{a(r)+\sum_{i} \lambda_{i}}
\end{aligned}
$$

By induction on the height of the tree, we can assume

$$
E U\left(H\left(z_{i}, s_{i}\right)\left(y^{\equiv}, r\right)\right)=E u\left(H\left(z_{i}\right) \mid r\right)
$$

Then the prior equation becomes

$$
\begin{aligned}
& E U\left(H\left(y^{\equiv}, r\right)\left(\left(x^{\equiv}, q\right)\right)\right. \\
& =w(r \mid q)+\Delta w(r \mid q) \frac{v(r)+\sum_{i} \lambda_{i} E u\left(H\left(z_{i}\right) \mid r\right)}{a(r)+\sum_{i} \lambda_{i}} \\
& =E u\left(H_{y} \mid q\right)
\end{aligned}
$$

For chance forks $H$ the proof is similar.

\section{Equivalence of Markovian utility functions}

Lemma 1: Two Markovian utility functions $u$ and $u^{\prime}$ with $u(\varnothing)=u^{\prime}(\varnothing)=0$ are strategically equivalent if and only if there is a positive constant $c$ such that

$$
\begin{array}{ll}
w^{\prime}\left(x \mid x_{0}\right)=c w\left(x \mid x_{0}\right) & \Delta w^{\prime}\left(x \mid x_{0}\right)=\Delta w\left(x \mid x_{0}\right) \\
v^{\prime}(x)=c v(x) & a^{\prime}(x)=a(x)
\end{array}
$$

Proof: Because $u$ and $u^{\prime}$ share a common zero point, strategic equivalence forces $u^{\prime}=c u$ for some positive $c$. Consider a stochastic tree $x^{\lambda} K$. Given Markovian preference state $x_{0}$, expected utilities are

$$
\begin{aligned}
& E\left[u\left(x^{\lambda} K \mid x_{0}\right)\right]=w\left(x \mid x_{0}\right)+\Delta w\left(x \mid x_{0}\right) \cdot(v(x) m(a(x)+\lambda) \\
& \quad+(1-a(x) m(a(x)+\lambda)) E[u(K \mid x)]) \\
& E\left[u^{\prime}\left(x^{\lambda} K \mid x_{0}\right)\right]=w^{\prime}\left(x \mid x_{0}\right)+\Delta w^{\prime}\left(x \mid x_{0}\right)\left(v^{\prime}(x) m\left(a^{\prime}(x)+\lambda\right)\right. \\
& \left.\quad+\left(1-a^{\prime}(x) m\left(a^{\prime}(x)+\lambda\right)\right) E\left[u^{\prime}(K \mid x)\right]\right)
\end{aligned}
$$

Set $K=\varnothing$ in the above to get

$$
E\left[u\left(x^{\lambda} \mid x_{0}\right)\right]=w\left(x \mid x_{0}\right)+\Delta w\left(x \mid x_{0}\right) \cdot v(x) m(a(x)+\lambda)
$$$$
E\left[u^{\prime}\left(x^{\lambda} \mid x_{0}\right)\right]=w^{\prime}\left(x \mid x_{0}\right)+\Delta w^{\prime}\left(x \mid x_{0}\right) v^{\prime}(x) m\left(a^{\prime}(x)+\lambda\right)
$$

Then $u^{\prime}=c u$ implies

$$
\begin{aligned}
& w^{\prime}\left(x \mid x_{0}\right)+\Delta w^{\prime}\left(x \mid x_{0}\right) v^{\prime}(x) m\left(a^{\prime}(x)+\lambda\right) \\
& \quad=c\left(w\left(x \mid x_{0}\right)+\Delta w\left(x \mid x_{0}\right) \cdot v(x) m(a(x)+\lambda)\right)
\end{aligned}
$$

Set $\lambda=\infty$ to get 


$$
w^{\prime}\left(x \mid x_{0}\right)=c w\left(x \mid x_{0}\right)
$$

Substitute this back into its predecessor and cancel to get

$$
\begin{aligned}
& \Delta w^{\prime}\left(x \mid x_{0}\right) v^{\prime}(x) m\left(a^{\prime}(x)+\lambda\right) \\
& \quad=c \Delta w\left(x \mid x_{0}\right) \cdot v(x) m(a(x)+\lambda)
\end{aligned}
$$

Let $\lambda(t)$ be a constant $\lambda$ to conclude

$$
\begin{aligned}
& \Delta w^{\prime}\left(x \mid x_{0}\right) v^{\prime}(x) /\left(a^{\prime}(x)+\lambda\right) \\
& =c \Delta w\left(x \mid x_{0}\right) \cdot v(x) /(a(x)+\lambda)
\end{aligned}
$$

The equality of two functions $k^{\prime} /\left(a^{\prime}+\lambda\right)$ and $k /(a+\lambda)$ of $\lambda \in[0, \infty)$ forces $k^{\prime}=k$ and $a^{\prime}=a$. Therefore we conclude

$$
\begin{aligned}
& \Delta w^{\prime}\left(x \mid x_{0}\right) v^{\prime}(x)=c \Delta w\left(x \mid x_{0}\right) \cdot v(x) \\
& a^{\prime}(x)=a(x) .
\end{aligned}
$$

Substitute (3),(4),(5) back into (2), then use $u^{\prime}=c u$ and simplify to get

$$
\begin{gathered}
c E\left[u\left(x^{\lambda} K \mid x_{0}\right)\right]=c w\left(x \mid x_{0}\right)+c \Delta w\left(x \mid x_{0}\right) v(x) m(a(x)+\lambda) \\
+\Delta w^{\prime}\left(x \mid x_{0}\right)(1-a(x) m(a(x)+\lambda)) c E[u(K \mid x)]
\end{gathered}
$$

Substitute the right side of (1) for $E\left[u\left(x^{\lambda} K \mid x_{0}\right)\right]$ in the last equation and simplify to get

$$
\begin{aligned}
& \left.c \Delta w\left(x \mid x_{0}\right)(1-a(x) m(a(x)+\lambda)) E[u(K \mid x)]\right) \\
& \quad=\Delta w^{\prime}\left(x \mid x_{0}\right)(1-a(x) m(a(x)+\lambda)) c E[u(K \mid x)]
\end{aligned}
$$

Cancel terms to conclude $\Delta w\left(x \mid x_{0}\right)=\Delta w^{\prime}\left(x \mid x_{0}\right)$.

Therefore from (4) we conclude $v^{\prime}(x)=c v(x)$. We have thus shown that strategic equivalence of $u$ and $u^{\prime}$ imply the four equalities specified in Lemma 1.
Conversely, if these four equalities hold, then from (2) we obtain

$$
\begin{gathered}
E\left[u^{\prime}\left(x^{\lambda} K \mid x_{0}\right)\right]=c w\left(x \mid x_{0}\right)+\Delta w\left(x \mid x_{0}\right)\left(c v(x) m\left(a^{\prime}(x)+\lambda\right)\right. \\
\left.+\left(1-a^{\prime}(x) m\left(a^{\prime}(x)+\lambda\right)\right) E\left[u^{\prime}(K \mid x)\right]\right)
\end{gathered}
$$

Inducting on the height of the stochastic tree, if we have $E\left[u^{\prime}(K \mid x)\right]=c E[u(K \mid x)]$, then from (6) we conclude $E\left[u^{\prime}\left(x^{\lambda} K \mid x_{0}\right)\right]=c E\left[u\left(x^{\lambda} K \mid x_{0}\right)\right]$. Hence $u^{\prime}$ and $u$ are strategically equivalent.

\section{Proof of Theorem 2}

Given Markovian utility over stochastic trees with product states $(y, z)$, define the following marginal functions:

$$
\begin{aligned}
& v_{l}(y)=v\left(y, z^{*}\right) \quad v_{2}(z)=v\left(y^{*}, z\right) \\
& a_{1}(y)=a\left(y, z^{*}\right) \quad a_{2}(z)=a\left(y^{*}, z\right) \\
& w_{l}\left(y \mid y^{\prime}\right)=w\left(y, z^{*} \mid y^{\prime}, z^{*}\right) \\
& w_{2}\left(z \mid z^{\prime}\right)=w\left(y^{*}, z \mid y^{*}, z^{\prime}\right) \\
& \Delta w_{l}\left(y \mid y^{\prime}\right)=\Delta w\left(y, z^{*} \mid y^{\prime}, z^{*}\right) \\
& \Delta w_{2}\left(z \mid z^{\prime}\right)=\Delta w\left(y^{*}, z \mid y^{*}, z^{\prime}\right)
\end{aligned}
$$

and let $v^{*}=v\left(y^{*}, z^{*}\right)$. Suppose non-interfering utility independence holds. Consider a stochastic tree $\left(y^{\lambda} K, z\right)$. Its Markovian expected utility given preference summary $\left(y_{0}, z\right)$ is

$$
\begin{gathered}
E\left[u\left(y^{\lambda} K, z \mid y_{0}, z\right)\right]=w\left(y, z \mid y_{0}, z\right)+\Delta w\left(y, z \mid y_{0}, z\right) \\
(v(y, z) m(a(y, z)+\lambda)+(1-a(y, z) m(a(y, z)+\lambda)) \\
E[u(K, z \mid y, z)])
\end{gathered}
$$

Substitute $z=z^{*}$ to get 


$$
\begin{gathered}
E\left[u\left(y^{\lambda} K, z^{*} \mid y_{0}, z^{*}\right)\right]=w_{l}\left(y \mid y_{0}\right)+\Delta w_{l}\left(y \mid y_{0}\right) \\
\left(v_{l}(y) m\left(a_{l}(y)+\lambda\right)+\left(1-a_{l}(y) m\left(a_{l}(y)+\lambda\right)\right)\right. \\
\left.E\left[u\left(K, z^{*} \mid y, z^{*}\right)\right]\right)
\end{gathered}
$$

Non-interfering utility independence of factor 1

from factor 2 implies the last two expressions are equivalent Markovian utility functions over factor

1. Invoking Lemma 1, we conclude there is a positive $c_{2}(z)$ such that

$$
\begin{array}{ll}
v(y, z)=v_{1}(y) c_{2}(z) & a(y, z)=a_{1}(y) \\
w\left(y, z \mid y_{0}, z\right)=w_{1}\left(y \mid y_{0}\right) c_{2}(z) & \Delta w\left(y, z \mid y_{0}, z\right)=\Delta w_{1}\left(y \mid y_{0}\right)
\end{array}
$$

Similarly, non-interfering utility independence of factor 2 from factor 1 implies there is a positive $c_{l}(y)$ such that

$$
\begin{array}{ll}
v(y, z)=c_{1}(y) v_{2}(z) & a(y, z)=a_{2}(z) \\
w\left(y, z \mid y, z_{0}\right)=c_{1}(y) w_{2}\left(z \mid z_{0}\right) & \Delta w\left(y, z \mid y, z_{0}\right)=\Delta w_{2}\left(\mathrm{z} \mid z_{0}\right)
\end{array}
$$

Repeatedly invoking these restrictions, we obtain condition (i) of the theorem:

$$
a(y, z)=a_{1}(y)=a\left(y, z^{*}\right)=a_{2}\left(z^{*}\right)=a^{*}
$$

In a similar way

$$
\begin{aligned}
v(y, z) & =v_{1}(y) c_{2}(z) \\
& =v\left(y, z^{*}\right) c_{2}(z) \\
& =c_{1}(y) v_{2}\left(z^{*}\right) c_{2}(z) \\
& =c_{1}(y) c_{2}(z) v^{*}
\end{aligned}
$$

Set $z=z^{*}$ in the first equality of this sequence to get $c_{2}\left(z^{*}\right)=1$. Similarly, $c_{l}\left(y^{*}\right)=1$. Substitute $z$ $=z$ into the last equality of the sequence to get $v_{l}(y)=c_{l}(y) v^{*}$. Similarly, $v_{2}(z)=c_{2}(z) v^{*}$.
Conditions (ii), (iii), (iv) of the theorem follow immediately.

Conversely, if conditions (i) - (iv) all hold, then (7) becomes

$$
\begin{gathered}
E\left[u\left(y^{\lambda} K, z \mid y_{0}, z\right)\right]=w_{l}\left(y \mid y_{0}\right) v_{2}(z) v^{*}+\Delta w_{l}\left(y \mid y_{0}\right) \\
\left(v_{l}(y) v_{2}(z) v^{*} m\left(a^{*}+\lambda\right)+\left(1-a^{*} m\left(a^{*}+\lambda\right)\right)\right. \\
E[u(K, z \mid y, z)]) \\
=\left(w_{l}\left(y \mid y_{0}\right)+\Delta w_{l}\left(y \mid y_{0}\right)\left(v_{l}(y) m\left(a^{*}+\lambda\right)\right) v_{2}(z) v^{*}\right. \\
\quad+\Delta w_{l}\left(y \mid y_{0}\right)\left(1-a^{*} m\left(a^{*}+\lambda\right)\right) E[u(K, z \mid y, z)]
\end{gathered}
$$

and (8) becomes

$$
\begin{aligned}
& E\left[u\left(y^{\lambda} K, z^{*} \mid y_{0}, z^{*}\right)\right]=w_{l}\left(y \mid y_{0}\right)+\Delta w_{l}\left(y \mid y_{0}\right) \\
& \left(v_{l}(y) m\left(a^{*}+\lambda\right)+\left(1-a^{*} m\left(a^{*}+\lambda\right)\right)\right. \\
& \left.E\left[u\left(K, z^{*} \mid y, z^{*}\right)\right]\right) \\
& =w_{l}\left(y \mid y_{0}\right)+\Delta w_{l}\left(y \mid y_{0}\right) v_{l}(y) m\left(a^{*}+\lambda\right) \\
& \quad+\Delta w_{l}\left(y \mid y_{0}\right)\left(1-a^{*} m\left(a^{*}+\lambda\right)\right) E\left[u\left(K, z^{*} \mid y, z^{*}\right)\right]
\end{aligned}
$$

We induct on the height of the stochastic tree. If the induction hypothesis

$$
E[u(K, z \mid y, z)]=E\left[u\left(K, z^{*} \mid y, z^{*}\right)\right] v_{2}(z) v^{*}
$$

holds for the subtree $K$ following $y$ then it follows from the last two equations that

$$
E\left[u\left(y^{\lambda} K, z \mid y_{0}, z\right)\right]=E\left[u\left(y^{\lambda} K, z^{*} \mid y_{0}, z^{*}\right)\right] v_{2}(z) v^{*}
$$

Therefore, by induction, the last equation holds for all states $y$ and subsequent subtrees $K$. Therefore factor 1 is non-interfering utility independent of factor 2, as claimed. Similarly, factor 2 is noninterfering utility independent of factor 1 . 


\section{UNUSABLE MATERIAL}

Marginal preference conditions

Even non-interfering utility independence is too strict, in that it forces $a(y, z)$ to be constant in $y, z$. Next we examine independence conditions under which $a(y, z)$ is not constant, but depends only on $a_{1}(y), a_{2}(z)$.

Assuming that risk attitude can be captured separately in each factor, an argument involving equivalent risk neutral preference presented in Figure 12 suggests that the factor risk-aversion coefficients $a_{1}(y)$ and $a_{2}(z)$ should combine additively to give $a(y, z)$.

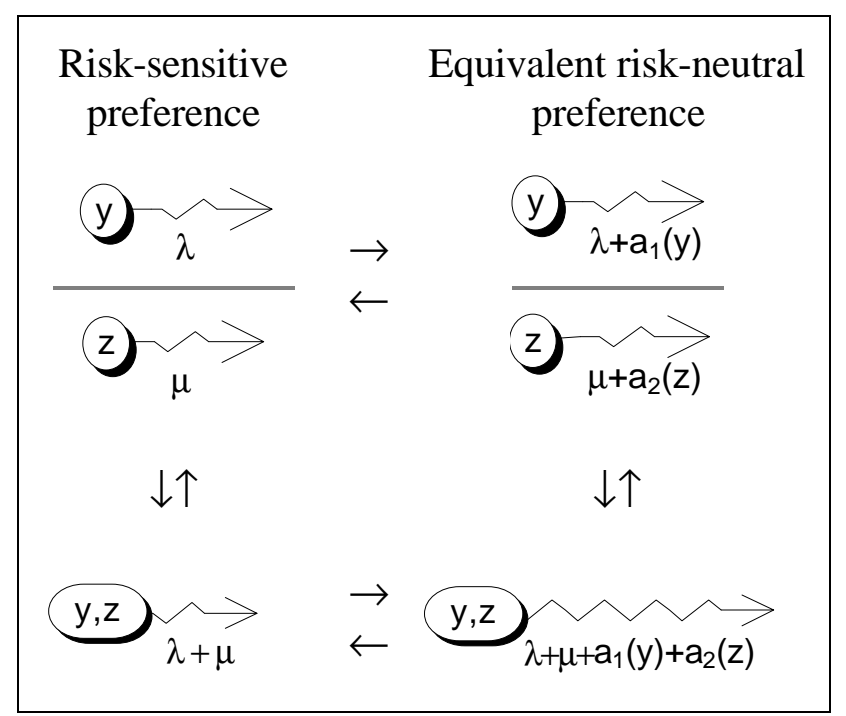

Figure 12: A heuristic argument involving riskneutral preference suggests that the coefficient of risk aversion $\mathrm{a}(\mathrm{y}, \mathrm{z})$ should decompose additively.

However, this argument is only heuristic. Preference conditions which yield an additive decomposition for $a(y, z)$ are given in the following theorem.

Theorem 3: Under Markovian utility, a necessary and sufficient condition for the relation

$$
a(y, z)=a_{1}(y)+a_{2}(z)
$$

is that

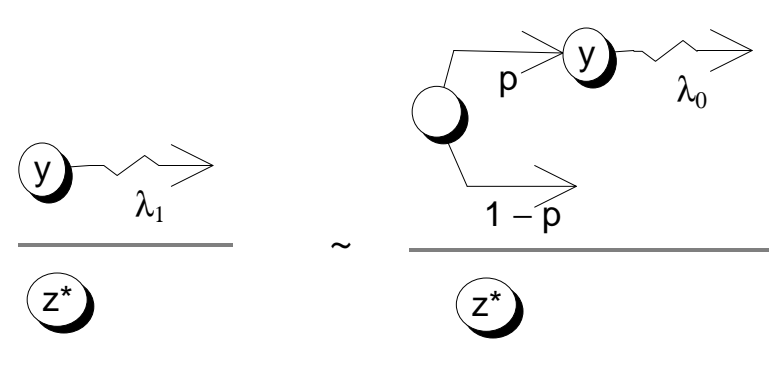

and

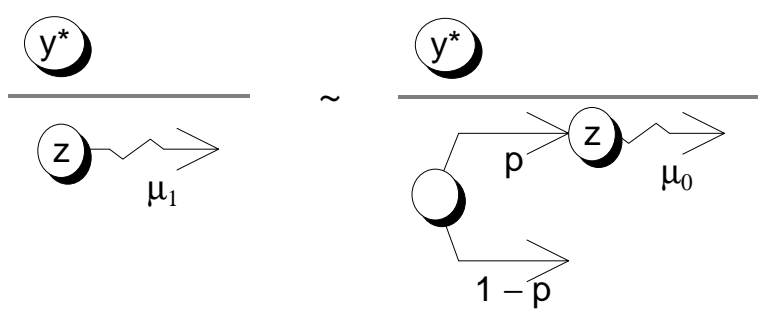

imply
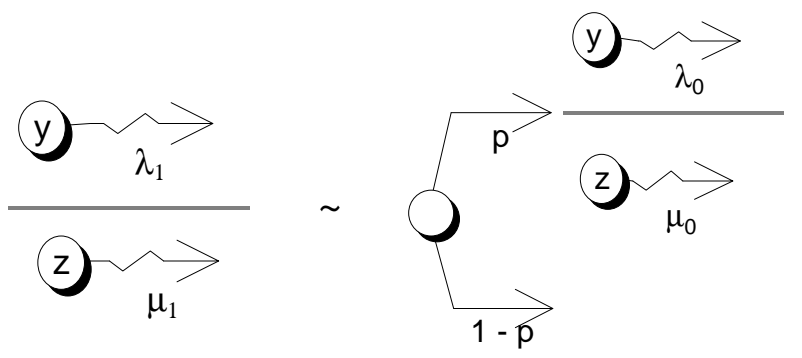

for all $\lambda_{l}, \lambda_{o}, \mu_{l}, \mu_{o}$. 
Theorem 3 is not as satisfying as one would wish because of the special role played by the distinguished states $y^{*}, z^{*}$. If the condition of Theorem 3 holds for $y^{*}, z^{*}$, then why should it not hold for any two states $y^{\prime}, z^{\prime}$ ? But this broader assumption yields

$$
a(y, z)=a\left(y, z^{\prime}\right)+a\left(y^{\prime}, z\right)
$$

for all states $y, z, y^{\prime}, z^{\prime}$, and from this it follows that $a(y, z)=0$ for all $y, z$. So caution is advised in the use of the condition of Theorem 3 . 